\title{
Comportamento assertivo e sua relação com ansiedade, locus de controle e auto-estima em estudantes universitários
}

\section{Assertive behavior and its relation to anxiety, locus of control and self esteem in undergraduate students}

\author{
Marina BANDEIRA' \\ Maria Amélia Cesari QUAGLIA' \\ Lívia da Silva BACHETTI' \\ Tatiana Lourençoni FERREIRA' \\ Grasiela Gomide de SOUZA'
}

\begin{abstract}
Resumo
Foram estudados o comportamento assertivo e sua relação com ansiedade, locus de controle e auto-estima. Participou da pesquisa uma amostra aleatória de 135 universitários, de ambos os sexos, com idade média de 25 anos. Foram aplicadas as escalas de assertividade de Rathus, locus de controle de Levenson, auto-estima de Dela Coleta e a escala de ansiedade IDATE-traço. Os resultados indicaram que os estudantes da área de ciências exatas apresentaram escores mais elevados de assertividade e de auto-estima e escores menos elevados de ansiedade do que os de ciências humanas. Não houve diferença significativa de gênero. Os resultados mostraram uma correlação positiva significativa entre os escores de assertividade e o grau de internalidade $(r=0,23)$ e de auto-estima $(r=0,54)$. Constatou-se ainda uma correlação negativa significativa entre assertividade e grau de ansiedade $(r=-0,55)$. Esses resultados confirmam dados da literatura, indicando que o comportamento assertivo está relacionado à baixa ansiedade e à alta internalidade e à alta auto-estima, com implicações para o treinamento do comportamento assertivo.
\end{abstract}

Palavras-chave: ansiedade; auto-estima; comportamento assertivo; locus de controle.

\begin{abstract}
The assertive behavior and its relation to the anxiety, locus of control and selfesteem have been evaluated. This study sample was random by selected and composed by 135 undergraduate students, 25 years old in average, male or female. The subjects had filled in the following scales: Rathus' assertive scale, Levensons' locus of control scale, IDATE-trace anxiety scale and Dela Coleta self esteem scale. Results have indicated that students from Science area presented higher assertiveness and self esteem scores, and lower anxiety scores than students from Social Sciences. No gender differences were observed. Results showed that the assertive behavior was positively correlated with internal locus of control $(r=0.23)$ and positive self esteem $(r=0.54)$. It was also obtained a significant negative correlation between assertiveness and anxiety $(r=0.055)$. These results replicated data from other researches, indicating the importance of a low level of anxiety and high level of internality and self esteem for assertive behavior and its implication for social skills training.
\end{abstract}

Key words: anxiety; selfesteem; assertive behavior; locus of control.

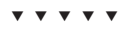

1 Laboratório de Pesquisa em Saúde Mental, Universidade Federal de São João del Rei. Praça Dom Helvécio, 74, Salas 2.06 e 2.10, Bairro Dom Bosco, 36301-160, São João del Rei, MG, Brasil. Correspondência para/Correspondence to: M. BANDEIRA. E-mail: <bandeira@ufjj.edu.br>. 
O conceito de assertividade, compreendido como uma subárea das habilidades sociais, envolve a afirmação dos próprios direitos e a expressão de pensamentos, sentimentos e crenças de maneira direta, honesta e apropriada, de modo a não violar o direito das outras pessoas (Lange \& Jakubowski, 1978). Tem sido estudado o papel de diversas variáveis em relação ao desempenho social, em particular o comportamento assertivo, tais como ansiedade, auto-estima e locus de controle, em uma tentativa de explicar as dificuldades apresentadas pelas pessoas em situações sociais.

Um dos modelos que explicam os déficits de desempenho social desenvolveu-se a partir das pesquisas de Wolpe (1958) sobre a neurose experimental com animais, baseando-se no paradigma do condicionamento respondente ou clássico (Del Prette \& Del Prette, 1999). Segundo esse modelo, conhecido como a teoria da inibição recíproca, os déficits de desempenho social, em particular a assertividade, poderiam ser explicados pelo efeito inibidor da ansiedade. Essa explicação se baseou no princípio fisiológico segundo o qual respostas antagônicas não poderiam ser emitidas simultaneamente (Wolpe, 1981). Uma das respostas que demonstrou ser competitiva com a assertividade foi a ansiedade. Segundo esse modelo, há um déficit de desempenho social quando situações sociais previamente emparelhadas com estímulos aversivos eliciam ansiedade no indivíduo, inibindo as respostas assertivas (Del Prette \& Del Prette, 1999). Esse modelo derivou a hipótese de que haveria uma correlação negativa entre a assertividade e a ansiedade, o que tem sido confirmado por diversas pesquisas. Por exemplo, Orenstein, Orenstein e Carr (1975), comparando três grupos de sujeitos com diferentes graus de assertividade, segundo seus escores na escala de Rathus (1973), mostraram que a assertividade relacionou-se inversa e significativamente com as variáveis neuroticismo, ansiedade-traço e

ansiedade interpessoal, para ambos os sexos. Pachman

Z e Foy (1978) observaram igualmente que a ansiedade era incompatível com o comporta-mento assertivo, em uma pesquisa com uma amostra de 55 pacientes alcoolistas do sexo masculino, utilizando a observação do comportamento em situações de desempenho de

112 papéis para avaliar o grau de assertividade.
Anderson (1997) observou também, em situações de desempenho de papéis, que sujeitos com maior grau de ansiedade, medida através de escalas, apresentavam um desempenho interpessoal inferior em termos de comportamentos assertivos verbais e não-verbais, tanto em situações de alto quanto de baixo nível de avaliação por outras pessoas. Na pesquisa de Creed e Funder (1998), com uma amostra de 149 estudantes universitários de ambos os sexos, também foi constatada essa mesma relação inversa, uma vez que os indivíduos socialmente ansiosos se avaliavam e eram avaliados pelos seus pares como inassertivos, além de apresentarem um déficit de habilidades sociais em interações sociais através de desempenho de papéis, comparativamente aos sujeitos não ansiosos.

Halford e Foddy (1982), estudando uma amostra de 45 universitários, constataram igualmente que sujeitos altamente ansiosos se auto-avaliavam e eram avaliados por juízes como menos assertivos do que os sujeitos com grau moderado e baixo de ansiedade, em situações de desempenho de papéis, além de apresentarem mais cognições associadas às reações negativas dos outros.

Segundo Schwartz e Gottman (1976), o principal déficit dos indivíduos inassertivos se situa no nível cognitivo. Em sua pesquisa, os sujeitos que haviam apresentado baixa assertividade, em situações de desempenho de papéis, relataram sentir um maior grau de tensão e possuir mais auto-avaliações negativas do que os sujeitos com moderada e alta assertividade, apesar de não haver diferenças entre eles quanto ao nível de ativação fisiológica e conhecimento do comportamento adequado a ser emitido. Os autores destacaram a importância da interpretação que os sujeitos fizeram a respeito das suas próprias reações fisiológicas. Tomaka (1999) constatou igualmente que sujeitos com baixo grau de assertividade apresentavam respostas cognitivas e fisiológicas diferentes de sujeitos com alto grau de assertividade, frente a situações estressantes de falar em público de improviso. Os mais assertivos consideraram essa situação como um desafio e relataram menos stress e mais emoções positivas, enquanto que os menos assertivos consideraram a situação como uma ameaça e relataram mais stress e mais emoções negativas. 
Tentando explicar a relação entre ansiedade e assertividade, Kremer, Bringle e Cave (1980) investigaram o papel do medo do conflito interpessoal, comparando dois grupos de trinta estudantes universitárias com escores altos e baixos em asserti-vidade, diante de situações experimentais com alto e baixo nível de conflito. Os resultados mostraram que os sujeitos inassertivos apresentaram diminuição de ansiedade somente nas situações em que não foi confirmado o conflito esperado, enquanto que nas situações de alto conflito eles se mantiveram ansiosos, indicando assim a importância do medo do conflito como variável explicativa da ansiedade. Os sujeitos assertivos, por outro lado, se mantiveram com baixa ansiedade no decorrer das situações, independen-temente do grau de conflito presente.

Uma relação inversa se observa igualmente entre a ansiedade e o desempenho social, em geral. Por exemplo, na pesquisa longitudinal de Strahan (2002), foram estudadas a ansiedade social, as habilidades sociais e o desempenho acadêmico de estudantes universitários. Os estudantes com ansieda-de social apresentaram dificuldades significativamente maiores nas três áreas de habilidades sociais avaliadas: a capacidade verbal de se expressar socialmente, a sensibilidade social, que envolvia a habilidade de interpretar adequadamente a comunicação verbal dos outros, e controle social, que se referia à autoapresentação e ao desempenho de papéis. Uma correlação negativa significativa entre habilidades sociais e grau de ansiedade social também foi encontrada na pesquisa de Riggio, Throckmorton e DePaola (1990), com uma amostra de 121 estudantes universitários avaliados através de escalas de autorelato. No caso particular da fobia social, Stopa e Clark (1993) também observaram uma relação inversa entre grau de ansiedade e o desempenho social, comparando três grupos: fóbicos sociais, indivíduos ansiosos e um grupo-controle. Os sujeitos fóbicos apresentaram um desempenho social inferior aos outros grupos, bem como pensamentos auto-avaliativos negativos em situações de conversação.

O desempenho social e o comportamento assertivo têm sido relacionados também a uma outra variável: que consiste no locus de controle. Trata-se de um construto que diz respeito a uma tendência relativamente estável das pessoas em explicar a causalidade dos eventos que ocorrem em suas vidas em termos de um contínuo entre internalidade e externalidade. Internalidade se refere a explicações em que o sujeito percebe a fonte de controle dos eventos de sua vida como de sua própria responsabilidade. Externalidade envolve explicações nas quais a fonte de controle dos eventos está localizada fora do sujeito (Dela Coleta, 1979; Dela Coleta, 1987). Esse construto tem sido avaliado através da escala de Rotter (1966) e da escala de Levenson (1973).

Algumas pesquisas têm constatado que o locus de controle interno estaria positivamente relacionado ao comportamento assertivo. Por exemplo, Appelbaum e Tuma (1975), em um estudo com 158 universitários de ambos os sexos, investigaram a relação entre locus de controle, desejabilidade social e assertividade, avaliada por meio de três escalas. Foi obtida uma correlação positiva significativa entre a assertividade e o locus de controle interno. Indivíduos com atribuição interna de causali-dade relataram mais comportamentos assertivos do que aqueles com orientação externa, independen-temente do grau de desejabilidade social. Do mesmo modo, Williams e Stout (1985) também observaram uma relação significativa entre assertividade, locus de controle e stress a partir de uma amostra de 78 universitários, comparando sujeitos com altos e baixos escores de assertividade. Os autores constataram que os indivíduos mais assertivos eram mais orientados internamente e apresentavam menor freqüência de sintomas de doenças relacionadas ao stress do que os indivíduos com um índice baixo de assertividade.

Molinari e Khanna (1981) colocaram em destaque a relação entre internalidade e bem-estar psicológico, uma vez que os indivíduos internos teriam uma atitude mais ativa e mais responsável em relação aos eventos de sua vida, seriam mais capazes de retardar as gratificações e de usar estratégias cognitivas eficazes, podendo assim apresentar uma tendência menor de desenvolver distúrbios psicopatológicos. É possível que a internalidade se relacione particular-mente com o comportamento assertivo e menos com as habilidades 
sociais em geral, pois na pesquisa de Riggio et al. (1990), por exemplo, não foi encontrada correlação significativa entre locus de controle e habilidades sociais em geral, tal como avaliada por escalas de auto-relato.

A auto-estima tem sido igualmente relacionada com o desempenho social, uma vez que ela pode influenciar a expectativa que as pessoas possuem sobre o resultado de seu próprio desempenho. Essa expectativa, por sua vez, tem sido considerada uma variável importante para o desempenho social, em particular do comportamento assertivo (Del Prette \& Del Prette, 1999). O conceito de auto-estima relacionase com uma tendência relativamente estável de sentir-se bem ou mal a respeito de si mesmo. A relação entre auto-estima e desempenho social foi investigada na pesquisa de Riggio et al. (1990), com uma amostra de 121 estudantes universitários, de ambos os sexos, avaliados através de escalas de auto-relato. Observou-se uma correlação positiva significativa entre as habilidades sociais e o grau de auto-estima. Esse mesmo resultado foi obtido em uma outra pesquisa (Riggio, Watring \& Throckmorton, 1993), com uma amostra de 136 estudantes universitários, usando escalas de auto-relato. Essas variáveis foram também preditivas de maior suporte social, maior satisfação com a vida acadêmica e com a vida em geral e menor percepção de solidão. A auto-estima pode afetar também o desempenho social das pessoas ao responderem ao feedback dos outros. Por exemplo, Kernis, Cornell, Sun, Berry e Harlow (1993), em um estudo com 125 universitários, observaram que indivíduos com alto grau de auto-estima aceitavam melhor o feedback positivo dos outros do que os indivíduos com baixa auto-estima. Ao contrário, os indivíduos com baixa auto-estima aceitavam melhor o feedback negativo dos outros do que os indivíduos com alta auto-estima.

Embora haja evidências na literatura interna(\%) cional de que as variáveis de ansiedade, locus de

z controle interno e auto-estima estejam relacionadas com o comportamento assertivo, não foram encontra-das pesquisas sobre esse tema no contexto brasileiro. O objetivo do presente trabalho consiste em preencher essa lacuna, investigando se o comportamento assertivo está relacionado com menor grau de ansiedade, maior grau de internalidade e maior auto-estima.

\section{Método}

Foram selecionados aleatoriamente quinze estudantes universitários matriculados nos dois últimos anos de cada um de nove cursos, do turno noturno, da Universidade Federal de São João del Rei. Os sujeitos estavam regularmente matriculados nos cursos de Administração, Ciências, Economia, Engenharia Industrial Elétrica, Engenharia Industrial Mecânica, Filosofia, Letras, Pedagogia e Psicologia. Os sujeitos foram selecionados a partir da lista de alunos incluídos nos diários de classe de cada curso. Partindo-se dessas listas, os sujeitos foram selecionados ao acaso, por meio de sorteio, o que caracterizou uma amostra aleatória simples.

Do total da amostra, 75 alunos pertenciam aos cursos da área de Ciências Exatas e 60 aos cursos da área de Ciências Humanas. A amostra consistiu de 135 sujeitos, na faixa etária entre 21 e 44 anos, com idade média de 25,49 anos ( $d p=4,55$ ), sendo 82 sujeitos do sexo masculino e 53 do sexo feminino.

\section{Instrumentos de medida}

Escala de assertividade: O grau de assertividade dos sujeitos foi medido através da escala de assertividade de Rathus (RAS) (Rathus, 1973). Trata-se de um instrumento de auto-avaliação, composto de 30 questões que medem o comportamento assertivo em situações sociais. Os sujeitos devem optar por uma dentre seis alternativas de resposta, dispostas em uma escala que se estende de "Extremamente parecido comigo" (+3) a "Extremamente diferente de mim"(-3).

A escala original foi traduzida e adaptada para o Brasil por Pasquali e Gouveia (1990), utilizando-se dois tradutores bilingües e uma avaliação semântica dos itens para avaliar sua compreensão. Foi feito um estudo de validação da escala para o contexto brasileiro, utilizando-se uma amostra de 302 estudantes secundaristas e universitários, de ambos os sexos, da faixa etária entre 12 e 52 anos. Foi verificada a sua validade através da análise fatorial, que indicou a presença de um fator nomeado inibição-desinibição. 
Para testar a fidedignidade do instrumento, os autores utilizaram a análise estatística Alpha de Cronbach, tendo sido obtido um valor de alfa de 0,81, indicando assim uma adequada consistência interna dos itens da versão brasileira da escala RAS.

Inventário de locus de controle de Levenson: Este instrumento foi utilizado para avaliar o tipo de atribuição dos sujeitos para explicar as causas dos eventos de sua vida (Dela Coleta, 1987). Trata-se de um instrumento de auto-avaliação, composto de 24 itens em que o sujeito deve optar entre alternativas de atribuição interna ou externa. O sujeito apresenta maior grau de locus de controle interno quando se percebe como causador e controlador dos eventos. Ao contrário, apresenta maior grau de locus de controle externo quando percebe que forças externas dominam e controlam os eventos de sua vida. O inventário é dividido em três escalas, cada uma delas compostas de oito questões. A escala 1 se refere a causas internas, a escala $P$ se refere ao efeito contro-lador de pessoas poderosas e a escala $C$ se refere a fatores do acaso. Quanto maior o escore obtido pelo sujeito em uma dessas escalas, mais próxima a crença do sujeito naquela fonte como controladora dos eventos de sua vida. Trata-se de escalas tipo Likert, em que os sujeitos devem optar por uma entre cinco alternativas, que se dispõem entre "Concordo totalmente" (1) e "Discordo totalmente" (5).

O inventário de locus de controle de Levenson foi traduzido e validado para o contexto brasileiro por Dela Coleta (1987). O instrumento foi aplicado em três amostras compostas por 188 estudantes universitários, 387 estudantes secundários e 100 adultos. Para verificar a validade discriminante da escala, utilizou-se a análise de variância, obtendo-se diferenças significativas entre os três grupos de indivíduos na escala $P(F=11,6$, $p<0,01)$ e na escala $C(F=18,86, p<0,01)$. A escala da internalidade não apresentou diferenças significativas entre os grupos. A consistência interna da escala brasileira foi verificada através da correlação entre os escores dos itens pares e ímpares, tendo-se obtido um coeficiente de correlação significativo para cada subescala. O coeficiente Alpha de Cronbach foi também utilizado para verificar a consistência interna do instrumento, obtendo-se os valores de alpha de 0,50, 0,62 e 0,63, respectivamente, para as escalas I, P e
C. Além disso, a escala brasileira apresenta itens com alto poder discriminativo, indicado pela diferença significativa encontrada entre os $25 \%$ de sujeitos com os escores superiores e os 25\% de escores inferiores. Esses resultados confirmam as propriedades psicométricas adequadas da escala brasileira.

Escala de auto-estima: O grau de auto-estima dos estudantes universitários foi avaliado pela escala de auto-estima construída por Dela Coleta (1980). Trata-se de uma medida unidimensional da auto-estima, que possui quinze afirmações sobre as avaliações que o sujeito faz sobre si mesmo. Para cada afirmação, o sujeito escolhe uma dentre duas opções: concordo (C), ou discordo (D). O grau de auto-estima é fornecido através de um gabarito, que atribui um ponto para cada resposta em acordo e zero ponto para cada resposta em desacordo. A pontuação máxima é de quinze pontos e a mínima é de zero ponto.

Essa escala foi construída a partir dos itens de outras escalas utilizadas na literatura da área. A qualidade discriminativa dos itens da escala foi considerada adequada, pois a grande maioria apresentou altos coeficientes de correlação com os escores obtidos na escala como um todo. Os quinze itens com maiores escores de correlação foram selecionados para compor o instrumento de medida. Foi construída uma segunda versão do instrumento de medida, contendo alternativas de resposta dispostas em uma escala tipo Likert de cinco níveis. Para verificar a consistência interna dessa segunda versão, o instrumento foi aplicado em 258 vestibulan-dos, tendo sido feita a análise dos resultados através do coeficiente Alpha de Cronbach, que indicou uma adequada consistência interna, com o valor de alfa de 0,82

Inventário de ansiedade traço-estado: A ansiedade foi medida através do inventário de ansiedade traço-estado (IDATE) (Spielberger, Gorsuch \& Lushene,1970). O IDATE é um inventário de auto-avaliação utilizado para medir o estado e o traço de ansiedade. Na presente pesquisa foram utilizados apenas os vinte itens referentes ao traço de ansiedade, já que se refere a características não transitórias da personalidade do indivíduo. As questões do inventário apresentam quatro alternativas de resposta, de 1 (quase nunca) a 4 (quase sempre). 
O IDATE foi traduzido, adaptado e validado para o Brasil por Biaggio e Natalício (1979). Foi avaliada a consistência interna da versão brasileira da escala através do coeficiente alpha de Cronbach, tendo sido obtidos os valores de alfa de 0,93 para o sexo masculino e 0,87 para o sexo feminino, na escala ansiedade-traço. Além disso, com exceção do item 6, todas as correlações item-total para a escala ansiedade-traço variaram de 0,47 a 0,83 na amostra total (Biagio \& Natalício, 1979).

A estabilidade temporal do instrumento foi adequada, pois se obteve um coeficiente de correlação de 0,83 entre os escores do teste e do reteste para a escala ansiedade-traço. A validade concorrente da versão brasileira foi obtida através dos dados de sujeitos bilingües, que fizeram o teste na forma em português e em inglês, tendo sido obtida uma correlação de 0,77 para a escala de ansiedade-traço (Biagio \& Natalício, 1979).

\section{Procedimento}

Os sujeitos foram selecionados a partir das listas de alunos referentes aos últimos períodos de nove cursos da instituição. Os selecionados foram contatados pelos pesquisadores com o objetivo de lhes apresentar informações gerais sobre a pesquisa e solicitar sua participação. Os sujeitos apresentaram seu consentimento em participar da pesquisa, ocasião em que os interessados estabeleceram os dias e horários disponíveis para a administração dos testes.

Para a realização da coleta de dados, os participantes foram conduzidos individualmente ou em pequenos grupos de, no máximo, três alunos para uma sala do laboratório de pesquisa, em função da disponibilidade de horário para participação do estudo. Antes do início da sessão, os sujeitos recebiam instruções sobre como responder aos instrumentos de medida e foram informados de que não havia respostas certas nem erradas, mas que eles deveriam responder segundo suas próprias percepções, opiniões e sentimentos pessoais. Foi explicado ainda que os resultados individuais teriam sigilo total.

Após as instruções gerais, os sujeitos responderam aos seguintes instrumentos de medida, na

116 seguinte ordem: inventário de ansiedade traço-estado, inventário de locus de controle de levenson, escala de auto-estima de Dela Coleta e escala de assertividade Rathus. Foram dadas, aos participantes, instruções específicas necessárias à aplicação de cada escala. Os aplicadores se mantiveram disponíveis durante a aplicação dos testes para responder a dúvidas e assegurar que todas as questões fossem respondidas, evitando assim a ocorrência de respostas em branco.

Os resultados foram computados em termos das médias e desvios-padrão das respostas dos sujeitos aos itens de cada teste. No caso da escala de locus de controle, foram computadas também as médias de cada uma das quatro subescalas que compõem esse teste, assim como o grau de internalidade total. Esse último foi calculado a partir da seguinte fórmula utilizada por Dela Coleta (1987): I.T.=I - $\underline{P+C / 2}$.

\section{Resultados}

A Tabela 1 apresenta as médias globais e os desvios-padrão obtidos pelos sujeitos em relação às variáveis de assertividade, auto-estima, ansiedade e locus de controle. Para essa última variável, são apresentadas ainda as estimativas obtidas nas seguintes subescalas: locus de controle interno (I), locus de controle externo referente a pessoas poderosas $(P)$, locus de controle externo referente ao efeito do acaso (C) e grau de internalidade total (IT).

A média encontrada para assertividade foi de 5,80 . Os escores dos sujeitos situaram-se entre um mínimo de - 55 a um máximo de 61 pontos. Os escores da escala de assertividade podem variar ao longo de um contínuo de -90 a 90 pontos. Quanto mais a pontuação se localiza próxima de zero, mais assertivo é o sujeito. Por outro lado, pontuações próximas a -90 e 90 indicam, respectivamente, comportamento não-assertivo e assertivo com tendência agressiva. Os resultados obtidos para a presente amostra indicam, portanto, um valor mediano de assertividade.

Em relação à variável locus de controle, o valor médio dos escores obtidos para o locus de controle interno foi de 29,13 pontos. Para o locus de controle externo, obteve-se a média de 17,60 pontos para o efeito de pessoas poderosas e uma média de 17,52 pontos para o efeito do acaso. As pontuações das 
subescalas de locus de controle podem variar num contínuo entre 8 e 40 pontos. Uma pontuação próxima a oito pontos indica baixos níveis de internalidade ou externalidade, enquanto uma pontuação próxima a 40 indica altos níveis de internalidade ou externalidade do sujeito. Nesta pesquisa, os escores para locus de controle interno posicionaram-se entre 16 e 40 pontos, enquanto que o locus de controle externo apresentou pontuações entre 10 e 31 para pessoas poderosas e 10 a 33 para o efeito do acaso. A média obtida para o grau de internalidade total (IT) foi de 11,55 pontos.

Pode-se observar (Tabela 1) que a média dos escores de auto-estima foi de 11,02, e os resultados abrangeram toda a dimensão da escala, com um mínimo de 1, índice de menor escore de auto-estima, e um máximo de 15, índice de maior escore de auto-estima.

Para a variável ansiedade, obteve-se um escore médio de 45,49. Os escores da escala de ansiedade IDATE-traço podem variar num contínuo entre 20 e 80
Tabela 1. Médias e desvios-padrão dos escores de assertividade, auto-estima, ansiedade e dos indicadores de locus de controle interno, externo e total, para a amostra total.

\begin{tabular}{lcr}
\hline Variáveis & $\mathrm{n}$ & $\mathrm{M} \pm \mathrm{DP}$ \\
\hline Assertividade & 135 & $5,80 \pm 24,85$ \\
Locus interno (I) & 135 & $23,13 \pm 4,39$ \\
Pessoas poderosas (P) & 135 & $17,60 \pm 4,22$ \\
Acaso (C) & 135 & $17,52 \pm 4,57$ \\
Internalidade total (IT) & 135 & $11,55 \pm 6,63$ \\
Auto-estima & 135 & $11,02 \pm 3,08$ \\
Ansiedade & 135 & $45,49 \pm 6,11$ \\
\hline
\end{tabular}

I=internalidade; $\mathrm{C}=$ acaso; $\mathrm{P}=$ pessoas poderosas; $\mathrm{IT}=$ Internalidade total.

pontos, indicando menor e maior nível de ansiedade, respectivamente. Na presente pesquisa, os escores dos sujeitos situaram-se entre um mínimo de 31 e um máximo de 61 pontos.

Observa-se (Tabela 2) que não foram encontradas diferenças significativas entre os grupos do sexo masculino e feminino para os escores obtidos nas variáveis avaliadas. Conclui-se, dessa forma, que os

Tabela 2. Médias e desvios-padrão dos escores de assertividade, auto-estima, ansiedade e dos indicadores de locus de controle, em função do gênero.

\begin{tabular}{|c|c|c|c|c|c|c|c|}
\hline Variáveis & $\mathrm{n}$ & M & \pm & DP & $\mathrm{t}$ & $d f$ & $p$ \\
\hline Assertividade & & & & & 1,23 & 133 & 0,22 \\
\hline Sexo masculino & 53 & 9,06 & \pm & 2,95 & & & \\
\hline Sexo feminino & 82 & 3,69 & \pm & 2,95 & & & \\
\hline Internalidade (I) & & & & & 0,16 & 133 & 0,87 \\
\hline Sexo masculino & 53 & 29,21 & \pm & 4,11 & & & \\
\hline Sexo feminino & 82 & 29,08 & \pm & 4,58 & & & \\
\hline Externalidade: acaso (C) & & & & & $-0,71$ & 133 & 0,48 \\
\hline Sexo masculino & 53 & 17,87 & \pm & 3,54 & & & \\
\hline Sexo feminino & 82 & 18,34 & \pm & 4,08 & & & \\
\hline Externalidade: poderosos (P) & & & & & $-1,04$ & 133 & 0,30 \\
\hline Sexo masculino & 53 & 17,13 & \pm & 3,64 & & & \\
\hline Sexo feminino & 82 & 17,90 & \pm & 4,55 & & & \\
\hline Internalidade Total (IT) & & & & & 0,720 & 133 & 0,47 \\
\hline Sexo masculino & 53 & 12,06 & \pm & 5,43 & & & \\
\hline Sexo feminino & 82 & 11,26 & \pm & 6,74 & & & \\
\hline Auto-estima & & & & & 1,83 & 133 & 0,69 \\
\hline Sexo masculino & 53 & 11,62 & \pm & 0,35 & & & \\
\hline Sexo feminino & 82 & 10,63 & \pm & 0,37 & & & \\
\hline Ansiedade & & & & & $-1,33$ & 133 & 0,19 \\
\hline Sexo masculino & 53 & 44,62 & \pm & 5,51 & & & \\
\hline Sexo feminino & 82 & 46,05 & \pm & 6,43 & & & \\
\hline
\end{tabular}


homens não diferem das mulheres na presente amostra no que diz respeito ao grau de assertividade, nem em relação às características de atribuição de causalidade, de auto-estima e de ansiedade.

Pode-se observar (Tabela 3) uma diferença significativa $(p=0,03)$ entre as médias dos escores dos estudantes de ciências exatas $(X=10,00)$ e dos estudantes de ciências humanas $(X=0,55)$ para a variável assertividade. Observa-se, também, para a variável auto-estima, uma diferença significativa $(p=0,00)$ entre a média dos escores obtidos pelos estudantes de ciências exatas $(X=11,84)$ e dos estudantes de ciências humanas $(X=10,00)$.
Obteve-se ainda uma diferença significativa $(p=0,01)$ para o grau de ansiedade em função da área de formação acadêmica. Os estudantes de ciências humanas apresentaram um escore médio de ansiedade significativamente maior $(X=46,00)$ do que os estudantes da área de ciências exatas $(X=44,32)$. Por outro lado, não foram encontradas diferenças significativas para as variáveis relativas ao locus de controle, em função da área de formação.

Depreende-se dos dados acima que os estudantes de ciências exatas são mais assertivos, possuem maior escore de auto-estima e menor grau de ansiedade que os estudantes de ciências humanas.

Tabela 3. Médias e desvios-padrão dos escores de assertividade, auto-estima, ansiedade e dos indicadores de locus de controle, em função da área de formação: Ciências Exatas e Ciências Humanas.

\begin{tabular}{|c|c|c|c|c|c|c|c|}
\hline Variáveis & $n$ & M & \pm & DP & $\mathrm{t}$ & df & p \\
\hline Assertividade & & & & & 2,23 & 133 & $0,03^{*}$ \\
\hline Ciências Exatas & 75 & 10,00 & \pm & 22,11 & & & \\
\hline Ciências Humanas & 60 & 0,55 & \pm & 27,18 & & & \\
\hline Internalidade (I) & & & & & 0,43 & 133 & 0,67 \\
\hline Ciências Exatas & 75 & 29,28 & \pm & 3,91 & & & \\
\hline Ciências Humanas & 60 & 28,95 & \pm & 4,95 & & & \\
\hline Externalidade: acaso (C) & & & & & $-1,83$ & 133 & 0,69 \\
\hline Ciências Exatas & 75 & 17,60 & \pm & 3,55 & & & \\
\hline Ciências Humanas & 60 & 18,85 & \pm & 4,21 & & & \\
\hline Externalidade: pessoas poderosas (P) & & & & & $-1,44$ & 133 & 0,15 \\
\hline Ciências Exatas & 75 & 17,13 & \pm & 4,13 & & & \\
\hline Ciências Humanas & 60 & 18,18 & \pm & 4,29 & & & \\
\hline Internalidade total (IT) & & & & & 1,46 & 133 & 0,47 \\
\hline Ciências Exatas & 75 & 12,27 & \pm & 5,21 & & & \\
\hline Ciências Humanas & 60 & 10,70 & \pm & 7,29 & & & \\
\hline Auto-estima & & & & & 3,594 & 133 & $0,00^{* *}$ \\
\hline Ciências Exatas & 75 & 11,84 & \pm & 0,28 & & & \\
\hline Ciências Humanas & 60 & 10,00 & \pm & 0,45 & & & \\
\hline Ansiedade & & & & & $-2,54$ & 133 & $0,01^{*}$ \\
\hline Ciências Exatas & 75 & 44,32 & \pm & 5,61 & & & \\
\hline Ciências Humanas & 60 & 46,05 & \pm & 6,43 & & & \\
\hline
\end{tabular}

${ }^{* *} p<0,01 ;{ }^{*} p<0,05 ; \mid=$ internalidade; $\mathrm{C}=$ acaso; $\mathrm{P}=$ pessoas poderosas; $\mid \mathrm{T}=$ =internalidade total.

Tabela 4. Correlação de Pearson entre os escores de assertividade e os escores das variáveis ansiedade, auto-estima e dos indicadores de locus de controle interno (I), locus de controle externo. ( $\mathrm{P}=$ pessoas poderosas e $\mathrm{C}=$ acaso) e internalidade total (IT).

\begin{tabular}{lcccccc}
\hline & Internalidade (I) & $\begin{array}{c}\text { Externalidade: } \\
\text { poderosos }(\mathrm{P})\end{array}$ & $\begin{array}{c}\text { Externalidade: } \\
\text { acaso: (C) }\end{array}$ & $\begin{array}{c}\text { Internalidade } \\
\text { total (IT) }\end{array}$ & $\begin{array}{c}\text { Auto-estima } \\
\text { Ansiedade }\end{array}$ \\
\hline Assertividade & $0,23^{*}$ & $-0,33^{*}$ & $-0,25^{*}$ & 0,36 & $0,54^{*}$ \\
\hline
\end{tabular}

${ }^{*} p<0,01$. 
Pode-se observar (Tabela 4) uma correlação positiva significativa $(r=0,54 ; p=0,00)$ entre os escores de assertividade e de auto-estima. Também pode ser observada uma correlação positiva significativa ( $r=0,23$ e $p=0,00$ ) entre os escores de assertividade e locus de controle interno. Ou seja, quanto maior a assertividade, maior o grau de atribuição de causalidade interna. Por outro lado, obteve-se uma correlação negativa significativa $(r=0,55$ e $p=0,00)$ entre assertividade e ansiedade. Também foi encontrada uma correlação negativa significativa entre os escores de assertividade e o de locus de controle externo para a influência de pessoas poderosas $(r=0,33$ e $p=0,00)$ e para a influência do acaso $(r=0,25$ e $p=0,00)$.

\section{Discussão}

Os resultados obtidos na presente pesquisa confirmam a hipótese de que o comportamento assertivo esteja relacionado com as variáveis ansiedade, auto-estima e locus de controle, em concordância com os dados da literatura desta área (Williams \& Stout,1985; Kernis et al., 1993; Riggio et al., 1993; Anderson, 1997; Creed \& Funder, 1998; Strahan, 2002). Os limites do desenho de uma pesquisa correlacional nos permitem identificar a presença de fatores relacionados positivamente ou negativamente com o comportamento assertivo, mas nos impedem, entretanto, de precisar em que sentido aponta a relação entre essas variáveis.

Foi observada, por exemplo, uma relação inversa entre o comportamento assertivo e o grau de ansiedade, pois quanto maior era a ansiedade manifesta pelos estudantes, menor era a assertividade relatada, confirmando dados de outros pesquisadores nessa área (Wolpe \& Lazarus, 1966; Wolpe, 1973). Creed e Funder (1998), por exemplo, constataram que sujeitos ansiosos eram vistos por seus pares e se autoavaliavam como inassertivos, além de apresentarem baixa competência social, avaliada através de observação direta do comportamento, em situações interpessoais de desempenho de papéis. Esses mesmos resultados foram obtidos, em situações sociais semelhantes, por Halford e Foddy (1982), ao compararem três grupos de sujeitos com diferentes graus de ansiedade, porém tais situações foram avaliadas por observadores treinados. Pachman e Foy (1978) e Anderson (1997) também encontraram, em situações de desempenho de papéis, evidências de que a ansiedade era incompatível com a assertividade. Riggio et al. (1990), assim como Strahan (2002), obtiveram correlações negativas significativas entre habilidades sociais e ansiedade social, medidas através de escalas de auto-avaliação. Stopa e Clark (1993) observaram que sujeitos fóbicos apresentaram um desempenho social menos adequado avaliado por juízes, além de apresentarem mais freqüentemente auto-avaliações negativas em situações de conver-sação quando comparados com um grupo-controle. O medo do conflito interpessoal se mostrou um fator moderador importante na relação entre a ansiedade e o comportamento assertivo na pesquisa de Kremer et al. (1980).

Os resultados descritos acima sugerem que a ansiedade possa constituir um fator que interfere na emissão do comportamento assertivo, dando suporte ao modelo desenvolvido por Wolpe, com base no condicionamento respondente (Del Prette \& Del Prette, 1999). Segundo esse modelo, a ansiedade constituiria um fator inibidor do comportamento assertivo, sendo necessária uma intervenção terapêutica para diminuí-la, a fim de obter um aumento do comportamento das pessoas de expressar idéias, sentimentos e opiniões na defesa dos próprios direitos.

Uma outra relação significativa positiva observada na presente pesquisa envolveu o comportamento assertivo e o tipo de atribuição caracterizada como locus de controle, pois os sujeitos que apresentaram maior grau de internalidade na atribuição de causalidade dos eventos de suas vidas foram os mais assertivos e, contrariamente, os sujeitos que atribuíram mais a fatores externos (efeito do acaso ou de pessoas poderosas) as causas dos eventos de suas vidas foram os sujeitos menos assertivos. Esses resultados confirmam dados de outros pesquisadores; Williams e Stout (1985), por exemplo, comparando sujeitos com alto e baixo grau de assertividade, encontraram que os assertivos apresentavam uma atribuição mais interna de causalidade, além de apresentarem menos problemas de saúde relacionados ao stress. Appelbaum e Tuma (1975) encontraram, igualmente, que sujeitos com atribuição interna de 
causalidade apresentaram maior grau de assertividade, avaliado através da escala de Rathus, independentemente dos sujeitos apresentarem ou não desejabilidade social, como variável moderadora. Williams e Stout (1985) também constataram que as pessoas mais assertivas eram mais orientadas internamente do que as inassertivas, além de apresentarem menos doenças relacionadas ao stress. Esses resultados sugerem que a maneira como as pessoas interpretam as causas dos eventos de suas vidas constitui um fator que está relacionado com a emissão do comportamento assertivo. Atribuir a si próprio as causas dos eventos da vida poderia talvez ter um efeito facilitador do comportamento assertivo.

Foi observado ainda, na presente pesquisa, que quanto maior o grau de assertividade dos sujeitos, maior seu escore de auto-estima. Esse resultado confirma dados de outros pesquisadores a respeito do desempenho social. Por exemplo, Riggio et al. (1990) obtiveram uma correlação positiva significativa entre as habilidades sociais e a auto-estima. Esse mesmo resultado foi obtido em uma outra pesquisa (Riggio et al. 1993), sendo que as variáveis de auto-estima e habilidades sociais foram preditivas de menor sentimento de solidão, maior suporte social e maior satisfação com a vida acadêmica e com a vida em geral. Kernis et al. (1993) observaram igualmente que a auto-estima afetava a habilidade social de receber o feedback dos outros, pois as pessoas com escore mais elevado de auto-estima apresentaram melhor desempenho de receber feedback positivo e as pessoas com escore menos elevado de auto-estima aceitaram mais o feedback negativo. Esses resultados são consistentes com o modelo cognitivo-comportamental segundo o qual o auto-conceito e a auto-estima afetam o desempenho social (Del Prette \& Del Prette, 1999), assim como as auto-verbalizações negativas sobre si próprio ou seu próprio desempenho pode contribuir para a evitação e a fobia social (Rangé, 1994). Esses resultados colocam em destaque a importância do comportamento assertivo e sua relação com o bem-estar psicológico das pessoas.

\section{Conclusão}

Os resultados obtidos nesta pesquisa confirmaram, portanto, os dados da literatura internacional desta área, indicando que o desempenho interpessoal dos indivíduos em interações sociais, em particular o comportamento assertivo, estava relacionado com um baixo grau de ansiedade, assim como maior grau de internalidade e escore mais elevado de auto-estima. Essa replicação de resultados fornece uma base empírica necessária para generalizar as conclusões a respeito dos fatores que interferem no comportamento assertivo para o nosso contexto cultural, ampliando assim o conhecimento nesta área.

Pesquisas futuras poderão estender esta investigação para amostras diversificadas de sujeitos ou diferentes estratégias metodológicas. O conhecimento das variáveis relacionadas ao comportamento assertivo, destacadas na presente pesquisa, tem implicações ainda para o treinamento das habilidades sociais.

\section{Referências}

Anderson, R.T. (1997). Anxiety or ignorance: the determinants of interpersonal skill display. Dissertation Abstracts International: Section B: The Sciences and Engineering, 57 (9-B), 595-599.

Appelbaum, A., \& Tuma, J. (1975). Internal-external control and social assertiveness of subjects high and low in social desirability. Psychological Reports, 37 (1), 319-322.

Biaggio, A., \& Natalício, L. (1979). Inventário de Ansiedade Traço-Estado. Rio de Janeiro: Cento Editor de Psicologia Aplicada.

Creed, A., \& Funder, D. (1998). Social anxiety: from the inside and outside. Personality and Individual Differences, 25, 19-33.

Del Prette, Z.A., \& Del Prette, A. (1999). Psicologia das habilidades sociais: terapia e educação. 2. ed. Petrópolis: Vozes.

Dela Coleta, J.A. (1979). Escala de locus de controle internoexterno de Rotter: um estudo exploratório. Arquivos Brasileiros de Psicologia, 31 (4), 167-181.

Dela Coleta, J.A. (1980). Atribuições de causalidade em presos, cegos e amputados. Tese de doutorado, Fundação Getúlio Vargas, Rio de Janeiro.

Dela Coleta, M. (1987). Escala multidimensional de locus de controle de Levenson. Arquivos Brasileiros de Psicologia, 39 (2), 79-97.

Halford, K., \& Foddy, M. (1982). Cognitive and social skills correlates of social anxiety. British Journal Clinical Psycholody, 21 (Feb), 17-28.

Kernis, M.H., Cornell, D.P., Sun, C., Berry, A., \& Harlow, T. (1993). There's more to self-esteem than whether it is high or low: the importance of stability of self-esteem. 
Journal of Personality and Social Psychology, 65 (6), 1190-1204.

Kremer, J.F., Bringle, R.G., \& Cave, P. (1980). Anxiety and aggression of high and low assertive persons following conflict. Personality and Individual Differences, 1 (4), 355-361.

Lange, A.J., \& Jakuboviski, P. (1978). Responsible assertive behavior. Illinois: Research Press.

Levenson, H. (1973). Activism and powerful others. Journal Consulting Clinical Psychology, 41 (3), 397-404.

Molinari, V., \& Kanna, P. (1981). Locus of control and its relationship to anxiety and depression. Journal of Personality Assessment, 45 (3), 314-319.

Orenstein, H., Orenstein, E., \& Carr, J.E. (1975). Assertiveness and anxiety: correlational study. Journal Behavior Therapy Experimental Psychiatry, 6 (3), 203-207.

Pachman, J.S., \& Foy, D.W. (1978). A correlational investigation of anxiety, self-esteem and depression: New findings with behavioral measures of assertiveness. Journal of Behavior Therapy and Experimental Psychiatry, 9 (2), 97-101.

Pasquali, L., \& Gouveia, V. (1990). Escala de Assertividade Rathus - RAS: adaptação brasileira. Psicologia Teoria e Pesquisa, 6 (3), 233-249.

Rangé, B.P. (1994). Terapia cognitivo-comportamental da fobia social. Journal Brasileiro da Psiquiatria, 43 (6), 327-331.

Rathus, S.A. (1973). 30-Item schedule for assessing assertive behavior. Behavior Therapy, 4 (3), 398-406.

Riggio, R.E., Throckmorton, B., \& DePaola, S. (1990). Social Skills and Self-Esteem. Person Individ Diff, 11 (8), 799-804.

Riggio, R.E., Watring, K.P., \& Throckmorton, B. (1993). Social Skills, Social Support, and Psychosocial Adjustment. Person Individ Diff, 15 (3), 175-280.
Rotter, J.B. (1966). Generalized expectancies for internal versus external control of reinforcement. Psychology Monography, 80 (1), Whole No 609.

Schwartz, R.M., \& Gottman, J.M. (1976). Toward a task analisis of Assertive Behavior. Journal of Consulting and Clinical Psychology, 44 (6), 910-920.

Spielberger, C.D., Gorsuch, R.L., \& Lushene, R.E. (1970). Manual for the state-trait anxiety inventory. Palo Alto, CA: Consulting Psychology Press.

Strahan, E.Y. (2002). The effects of social anxiety and social skills on academic performance. Person Individ Diff, 34 (2), 347-366.

Stopa, L., \& Clark, D.M. (1993). Cognitive processes in social phobia. Behav Res Ther, 31 (3), 255-267.

Tomaka, J. (1999). Assertiveness predicts threat and challenge reactions to potential stress among women. Journal of Personality and Social Psychology, 76 (6), 1008-1021.

Williams, J., \& Stout, J. (1985). The effect of high and low assetiveness on locus of control and health problems. The Journal of Psychology, 119 (2),169-173.

Wolpe, J.S. (1958). Psychoterapy by reciprocal inhibition. Stanford: Standord University Press.

Wolpe, J., \& Lazarus, A.A. (1966). Behavior therapytechniques. Oxford: Pergamon Press.

Wolpe, J. (1973). The practice of behavior therapy. New York: Pergamon Press.

Wolpe, J. (1981). The Dichotomy Between Classical Condictioned and Cognitively Learned Anxiety. Journal of Behavior Therapy Experimental Psychiatry, $12(1), 35-42$.

Recebido para publicação em 12 de novembro de 2003 e aceito em 1 de março de 2005. 
